\title{
General unifying features of controlled quantum phenomena
}

\author{
Alexander Pechen, Constantin Brif, ${ }^{*}$ Rebing Wu, ${ }^{\dagger}$ Raj Chakrabarti, ${ }^{\ddagger}$ and Herschel Rabitz \\ Department of Chemistry, Princeton University, Princeton, New Jersey 08544, USA
}

\begin{abstract}
Many proposals have been put forth for controlling quantum phenomena, including open-loop, adaptive feedback, and real-time feedback control. Each of these approaches has been viewed as operationally, and even physically, distinct from the others. This work shows that all such scenarios inherently share the same fundamental control features residing in the topology of the landscape relating the target physical observable to the applied controls. This unified foundation may provide a basis for development of hybrid control schemes that would combine the advantages of the existing approaches to achieve the best overall performance.
\end{abstract}

PACS numbers: 03.65.-w, 02.30.Yy, 32.80.Qk, 82.50.Nd

Steering the dynamics of quantum systems by means of external controls is a central goal in many areas of modern science, ranging from the creation of selective molecular transformations to quantum information processing [1]. The control may be either coherent (e.g., a shaped laser pulse [2-6]) or incoherent (e.g., a tailored environment [7] or a sequence of quantum measurements [8]). Quantum control is proving to be successful in the laboratory for manipulating a broad variety of physical, chemical, and biologically relevant processes $[1,5,6]$.

Many seemingly distinct approaches have been developed for controlling quantum phenomena [9], including open-loop control (OLC) in which model-based designs are directly applied in experiments [3], adaptive feedback control (AFC) which is a measurement-guided closed-loop laboratory procedure including resetting the system to the initial state on each loop iteration [4-6], and real-time feedback control (RTFC) which involves quantum measurement back-action on the system upon traversing the loop $[10,11]$. Each of these control schemes has been argued to be operationally, and even physically, distinct from the others. This Rapid Communication shows that all such scenarios are unified on a fundamental level by the common character of the control landscape $[12,13]$ which relates the physical objective $J[c]$ to the control variables $c$. This general foundation makes it possible to extend the powerful landscape-based methods of optimality analysis, originally developed for OLC and AFC [12], to RTFC. Furthermore, one can envision combinations of such schemes [9], or even the prospect of new unanticipated ones, possibly arising in the future, all of which will share the same fundamental control landscape features.

The structure of the control landscape (i.e., its topology characterized by the nature of the local and global extrema) determines the efficacy of a search for optimal solutions to the posed control problem $[14,15]$. Searching for the global maximum of the objective function $J[c]$ in the laboratory would be significantly hindered by the existence of local maxima that

\footnotetext{
* Present address: Sandia National Laboratories, Livermore, CA 94550, USA. ${ }^{\dagger}$ Present address: Department of Automation and Center for Quantum Information, Tsinghua University, Beijing 100084, P.R. China.

${ }^{\ddagger}$ Present address: School of Chemical Engineering, Purdue University, West Lafayette, IN 47907, USA.
}

act as traps during the optimization procedure. Even stochastic optimization algorithms could be impeded by a high density of traps [16]. If the objective function has no traps, then, in principle, nothing lies in the way of reaching the global maximum (i.e., the best possible yield), except limited controls. This work establishes that a common landscape topology is shared by all quantum control schemes, leading to two general conclusions about controlling quantum phenomena. First, there are no local optima to act as traps on the landscape for a wide class of control problems, thereby enabling highly efficient searches for globally optimal controls. Second, for each particular objective $J$, there exists a single special control $c_{*}$ which is universally optimal for all initial states of a system; this result implies that $c_{*}$ provides inherent robustness to variations of the initial system's state. For practical applications, these generic fundamental properties can facilitate more flexible laboratory implementations of quantum control as well as guide the design of suitable algorithms for laboratory optimization [17].

The control landscapes for OLC and AFC were studied in recent works $[12-15,18]$. For closed quantum systems, a control objective $J$ can be cast as a function of unitary evolution operators. Assuming evolution-operator controllability (i.e., that any unitary evolution can be produced by the available controls), the landscapes were shown to have no traps for typical objectives, including the expectation value of an observable [12] and the fidelity of a unitary quantum gate [13]. The absence of landscape traps was also analyzed for closed systems in the space of actual controls [14]. The landscapes for OLC and AFC of open quantum systems can be studied by casting an objective as a function of completely positive, trace-preserving maps (i.e., Kraus maps [9, 19]) which describe the system evolution. Assuming Kraus-map controllability (i.e., that any Kraus map can be produced by the available controls), the trap-free landscape topology was established for open-system observable control [18].

The analysis of the control landscapes for RTFC is performed below to significantly extend the prior analysis [18] and provide a single complete framework unifying the landscapes for all quantum control schemes, including OLC, AFC, and RTFC. Two distinct approaches to RTFC of quantum systems are considered. The first one is based on feedback employing measurements of some quantum system output channel to guide a classical controller $[11,20,21]$. The 
other feedback approach is a quantum analog of Watt's flyball governor-a self-regulating quantum machine. In this scenario (called coherent RTFC), no measurements with a classical output signal are involved, and instead another quantum subsystem is employed to facilitate the control action, so that the evolution of the composite quantum system, consisting of the "plant" (the subsystem of interest) and "controller" (the auxiliary subsystem), is coherent [22, 23]. The performance of practical RTFC controllers (classical, quantum, or both) can be, in principle, optimized using AFC [9], possibly guided by an initial OLC design, further enhancing the significance of a universal landscape topology common to all quantum control approaches.

In RTFC based on selective measurements of a single quantum system, the outcome of each measurement is random. After averaging over the random processes corresponding to all possible measurement outcomes, feedback control produces Kraus-type evolution of the controlled system. In RTFC based on measurements of an ensemble of quantum systems, the ensemble average produces a Kraus map as well. In coherent RTFC, if the plant and controller are initially prepared in a product state, the evolution of the plant is once again represented by a Kraus map. Therefore, all of these incarnations of RTFC generate Kraus-type evolution. Arbitrary Kraus maps can be engineered, for example, by using a simple quantum measurement combined with coherent feedback actions [24]. Adopting a previous analysis [18], we show, under the Krausmap controllability assumption, that quantum control landscapes have no traps for all considered types of RTFC. We will start with basic definitions, then prove that each considered type of RTFC produces Kraus-type evolution, and, finally, use these results to determine the quantum control landscape topology. The conclusion of the Rapid Communication will then draw the analysis together for a unified control landscape formulation including OLC, AFC, and RTFC.

In practically important situations the evolution of an open quantum system can be represented by a Kraus map [9, 19]. For an $n$-level system, any such map $\Phi$ can be cast as the operator-sum representation (OSR) [19]: $\Phi(\rho)=$ $\sum_{\nu=1}^{L} K_{\nu} \rho K_{\nu}^{\dagger}$, where $\rho$ is the density matrix (a positive, unittrace $n \times n$ matrix) and $\left\{K_{\nu}\right\}_{\nu=1}^{L}$ is a set of Kraus operators (complex $n \times n$ matrices satisfying the trace-preserving condition $\sum_{\nu=1}^{L} K_{\nu}^{\dagger} K_{\nu}=\mathbb{I}$ ). The OSR is not unique: Any map $\Phi$ can be represented using infinitely many sets of Kraus operators. We denote the set of all Kraus maps by $\mathcal{K}_{n}$. The Krausmap description of open-system dynamics is very general and includes both Markovian and non-Markovian regimes [25].

A generalized quantum measurement with $N_{0}$ possible outcomes $\left\{O_{\alpha}\right\}\left(\alpha=1,2, \ldots, N_{0}\right)$ is characterized by a family of Kraus operators $\left\{K_{\alpha, \beta}\right\}$. We denote the set of outcomes and corresponding Kraus operators for a given quantum measurement as $O:=\left\{O_{\alpha}, K_{\alpha, \beta}\right\}$. In particular, the projective measurement of a Hermitian observable $\hat{O}=\sum_{\alpha} O_{\alpha} \Pi_{\alpha}$ with eigenvalues $O_{\alpha}$ and spectral projectors $\Pi_{\alpha}$ corresponds to the case $K_{\alpha, \beta}=\Pi_{\alpha} \delta_{\alpha, \beta}$. If the measurement starts at time $t$ and the system density matrix before the measurement is $\rho(t)$, then the probability of the measurement outcome $O_{\alpha}$ will be
$p_{\alpha}=\operatorname{Tr}\left[\sum_{\beta} K_{\alpha, \beta} \rho(t) K_{\alpha, \beta}^{\dagger}\right]$.

When a selective measurement of duration $\tau_{\mathrm{m}}$ is performed and the outcome $O_{\alpha}$ is observed, the density matrix evolves as $\rho(t) \rightarrow \rho_{\alpha}\left(t+\tau_{\mathrm{m}}\right)$, where

$$
\rho_{\alpha}\left(t+\tau_{\mathrm{m}}\right)=\frac{1}{p_{\alpha}} \sum_{\beta} K_{\alpha, \beta} \rho(t) K_{\alpha, \beta}^{\dagger} .
$$

If the measurement is nonselective (i.e., the measurement outcome is not observed), the corresponding evolution $\rho(t) \rightarrow$ $\rho\left(t+\tau_{\mathrm{m}}\right)$ will be the average over all possible measurement outcomes:

$$
\rho\left(t+\tau_{\mathrm{m}}\right)=\sum_{\alpha} p_{\alpha} \rho_{\alpha}\left(t+\tau_{\mathrm{m}}\right)=\sum_{\alpha, \beta} K_{\alpha, \beta} \rho(t) K_{\alpha, \beta}^{\dagger} .
$$

The evolution in Eq. (2) defines a Kraus map $\Omega_{O}: \rho(t) \rightarrow$ $\Omega_{O}[\rho(t)]=\rho\left(t+\tau_{\mathrm{m}}\right)$, which is completely determined by the measurement $O$.

In RTFC, the measurement (or, in another variation, the interaction with an auxiliary quantum "controller") alters the evolution of the quantum system at each feedback iteration. Thus, the same quantum system is followed in real time in the feedback loop. Implementing RTFC on the atomic or molecular scale appears to be a very challenging technical problem, but its practical realization promises to significantly improve the ability to stabilize and control quantum systems. Consider now RTFC of a single quantum system, where a discrete series of selective measurements is performed and each measurement is followed by a feedback action (continuous feedback can be treated as the limit of the discrete case, resulting in the same control landscape topology). For the discrete case, the $i$ th iteration of the feedback process consists of a measurement $O^{i}$ with possible outcomes $\left\{O_{\alpha}^{i}\right\}$ at time $t_{i}$, followed by a feedback action dependent on the measurement outcome (the measured observables and feedback actions can be distinct at different iterations). The feedback action may be generally represented by a Kraus map that depends on the measurement outcome (a special case of the feedback action is a unitary transformation corresponding to a coherent control). If at the $i$ th iteration the measurement outcome $O_{\alpha}^{i}$ is observed, then the feedback action (of duration $\tau_{\mathrm{f}}$ ) described by the Kraus map $\Lambda_{\alpha}^{i}$ will be applied to the system, so that the density matrix will evolve as $\rho\left(t_{i}\right) \rightarrow \rho_{\alpha}\left(t_{i}+\tau_{\mathrm{m}}+\tau_{\mathrm{f}}\right)=\Lambda_{\alpha}^{i}\left[\rho_{\alpha}\left(t_{i}+\tau_{\mathrm{m}}\right)\right]$, where $\rho_{\alpha}\left(t_{i}+\tau_{\mathrm{m}}\right)$ is of the form (1), with the corresponding probability $p_{\alpha}^{i}$. The map $\Lambda_{\alpha}^{i}$ also includes the free evolution and influence of the environment. The system evolution after one feedback iteration, averaged over all possible measurement outcomes, $\rho\left(t_{i}+\tau_{\mathrm{m}}+\tau_{\mathrm{f}}\right)=\sum_{\alpha} p_{\alpha}^{i} \rho_{\alpha}\left(t_{i}+\tau_{\mathrm{m}}+\tau_{\mathrm{f}}\right)$, is therefore given by the transformation $\rho\left(t_{i}\right) \rightarrow \rho\left(t_{i}+\tau_{\mathrm{m}}+\tau_{\mathrm{f}}\right)=\Phi^{i}\left[\rho\left(t_{i}\right)\right]$, where

$$
\Phi^{i}\left[\rho\left(t_{i}\right)\right]=\sum_{\alpha} \Lambda_{\alpha}^{i}\left[\sum_{\beta} K_{\alpha, \beta}^{i} \rho\left(t_{i}\right)\left(K_{\alpha, \beta}^{i}\right)^{\dagger}\right]
$$

and $\left\{K_{\alpha, \beta}^{i}\right\}$ are the Kraus operators characterizing the measurement $O^{i}$. Let the OSR of the feedback-action Kraus map 
$\Lambda_{\alpha}^{i}$ be $\Lambda_{\alpha}^{i}(\rho)=\sum_{\nu} L_{\nu, \alpha}^{i} \rho\left(L_{\nu, \alpha}^{i}\right)^{\dagger}$, where $\sum_{\nu}\left(L_{\nu, \alpha}^{i}\right)^{\dagger} L_{\nu, \alpha}^{i}=$ II. Then Eq. (3) can be rewritten as

$$
\Phi^{i}\left[\rho\left(t_{i}\right)\right]=\sum_{\nu, \alpha, \beta} Z_{\nu, \alpha, \beta}^{i} \rho\left(t_{i}\right)\left(Z_{\nu, \alpha, \beta}^{i}\right)^{\dagger},
$$

where $Z_{\nu, \alpha, \beta}^{i}=L_{\nu, \alpha}^{i} K_{\alpha, \beta}^{i}$. Since $\sum_{\nu, \alpha, \beta}\left(Z_{\nu, \alpha, \beta}^{i}\right)^{\dagger} Z_{\nu, \alpha, \beta}^{i}=$ $\mathbb{I}$, the transformation $\Phi^{i}$ of Eqs. (3) and (4) is a Kraus map. Thus, Eq. (4) shows that the average evolution for one feedback iteration is of the Kraus type for arbitrary (generalized) quantum measurement and any (coherent or incoherent) feedback action. For a special case of a pure, least-disturbing measurement and a coherent feedback action, this result was obtained in Ref. [24].

The entire feedback process for controlling the system from the initial to final time is characterized by a sequence of measurements and feedback actions: $F=$ $\left\{O^{1}, F^{1}, \ldots, O^{N}, F^{N}\right\}$, where $O^{i}(i=1,2, \ldots, N)$ is the measurement for the $i$ th iteration, $F^{i}=\left\{\Lambda_{\alpha}^{i}\right\}$ is the set of all feedback actions (Kraus maps) for the $i$ th iteration, and $N$ is the number of iterations. Different trials of the feedback process will, in general, produce distinct evolutions, resulting in different system states at the final time $T$. The average output of the feedback process is given by averaging over all possible evolutions, which produces the transformation $\rho(T)=\Phi_{F}[\rho(0)]$, where $\Phi_{F}=\Phi^{N} \circ \cdots \circ \Phi^{2} \circ \Phi^{1}$ is a Kraus map given by the composition of one-iteration Kraus maps of the form (4).

Consider now RTFC of an ensemble of identical quantum systems, where measurements record the expectation value $\bar{O}=\sum_{\alpha} p_{\alpha} O_{\alpha}=\operatorname{Tr}[\hat{O} \rho(t)]$ of an observable $\hat{O}$. The density matrix representing the state of the ensemble undergoes a transformation characteristic for a nonselective measurement (i.e., with averaging over all possible measurement outcomes): $\rho(t) \rightarrow \rho\left(t+\tau_{\mathrm{m}}\right)=\Omega_{O}[\rho(t)]$, where the Kraus map $\Omega_{O}$ is defined by Eq. (2). The feedback action conditioned upon the measured value $\bar{O}$ is generally represented by a Kraus map $\Lambda_{\bar{O}}$, so that the ensemble evolution for one feedback iteration is $\rho\left(t_{i}\right) \rightarrow \rho\left(t_{i}+\tau_{\mathrm{m}}+\tau_{\mathrm{f}}\right)=\Phi^{i}\left[\rho\left(t_{i}\right)\right]$, where the Kraus map $\Phi^{i}=\Lambda \frac{i}{O} \circ \Omega_{O}^{i}$ is the composition of the Kraus maps representing the ensemble measurement and feedback action for the $i$ th iteration. Similar to the singlesystem case, the overall transformation for the entire feedback process is $\rho(T)=\Phi_{F}[\rho(0)]$, where the Kraus map $\Phi_{F}=\Phi^{N} \circ \cdots \circ \Phi^{2} \circ \Phi^{1}$ is again the composition of oneiteration Kraus maps.

Consider now coherent RTFC, where the quantum subsystem of interest (the plant) interacts with an auxiliary quantum subsystem (the controller), and the evolution of the composite system is coherent: $\rho_{\text {tot }}(T)=U(T) \rho_{\text {tot }}(0) U^{\dagger}(T)$. Here, $\rho_{\text {tot }}$ and $U(T)$ are the density matrix and the unitary evolution operator, respectively, for the composite system. An external coherent control field (which is generally time-dependent) can act on the plant, controller, or both. The state of the plant at any time $t$ is represented by the reduced density matrix $\rho(t)=\operatorname{Tr}_{\mathrm{c}}\left[\rho_{\text {tot }}(t)\right]$, where $\operatorname{Tr}_{\mathrm{c}}$ denotes the trace over the controller's degrees of freedom. If the initial state of the composite system is in tensor product form, $\rho_{\text {tot }}(0)=\rho(0) \otimes \rho_{\mathrm{c}}(0)$, then the evolution of the plant is represented by a Kraus map: $\rho(T)=\Phi_{U}[\rho(0)]=\sum_{\nu} K_{\nu}^{U} \rho(0)\left(K_{\nu}^{U}\right)^{\dagger}$, where the Kraus operators $K_{\nu}^{U}$ depend on the evolution operator $U(T)$ of the composite system [19].

With the analysis above, we can now assess the topology of quantum control landscapes for all considered types of RTFC. To be specific, a prevalent problem in quantum control is to maximize the expectation value of some target observable of the controlled system. As we established above, in measurement-based RTFC for both a single quantum system and an ensemble, the average density matrix at the final time $T$ is given by $\rho(T)=\Phi_{F}[\rho(0)]$, where the Kraus map $\Phi_{F}$ is determined by the set $F$ of measurements and feedback actions at all iterations of the feedback process. The goal is to find an optimal feedback process $F_{\mathrm{opt}}$ that maximizes the expectation value of the target observable $\hat{A}$ at the final time, $\bar{A}(T)=\operatorname{Tr}[\hat{A} \rho(T)]$. The feedback process $F$ plays the role of a set of controls, and the objective function has the form: $J[F]=\operatorname{Tr}\left\{\hat{A} \Phi_{F}[\rho(0)]\right\}$. An OSR of the Kraus map $\Phi_{F}$ is given by $\Phi_{F}[\rho(0)]=\sum_{\nu} K_{\nu}^{F} \rho(0)\left(K_{\nu}^{F}\right)^{\dagger}$, where $\left\{K_{\nu}^{F}\right\}$ is a set of Kraus operators depending on the feedback process $F$. The objective $J[F]$ can be cast as a function of Kraus operators:

$$
J\left[\left\{K_{\nu}^{F}\right\}\right]=\operatorname{Tr}\left[\hat{A} \sum_{\nu} K_{\nu}^{F} \rho(0)\left(K_{\nu}^{F}\right)^{\dagger}\right] .
$$

We assume that the set $\mathcal{F}$ of all available feedback processes (i.e., all available measurements and feedback actions) is rich enough to produce all possible Kraus maps, i.e., that the system is Kraus-map controllable. For coherent RTFC, the plant also undergoes a Kraus-type evolution, $\rho(T)=\Phi_{U}[\rho(0)]$, and the control objective $\bar{A}(T)$ can also be cast as a function of the Kraus operators: $J\left[\left\{K_{\nu}^{U}\right\}\right]=\operatorname{Tr}\left[\hat{A} \sum_{\nu} K_{\nu}^{U} \rho(0)\left(K_{\nu}^{U}\right)^{\dagger}\right]$, which has the same form as Eq. (5). For a sufficiently large controller prepared initially in a pure state, evolution-operator controllability of the composite system is a sufficient condition for Kraus-map controllability of the plant [26].

Building on recent OLC and AFC-motivated analysis of control landscape topology [18] for a system that is Krausmap controllable, the objective function of the form (5) has no local maxima for any initial state $\rho(0)$ and any Hermitian operator $\hat{A}$. This result implies that quantum control landscapes for OLC, AFC, measurement-based RTFC, coherent RTFC, and combinations thereof, all have the same trap-free topology, provided that the available controls are sufficient to produce any Kraus map. Furthermore, all local extrema of the objective function of the form (5) are saddles [18] that can be easily evaded by a suitable algorithm guiding an ascent over the landscape. The trap-free control landscape topology is established above in the space of Kraus maps, $\mathcal{K}_{n}$. The control landscape also will be trap-free in the space of actual controls, $\mathcal{C}$, if the tangent map from $\mathcal{C}$ to $\mathcal{K}_{n}$ is surjective everywhere in $\mathcal{C}$. Although, in general, there exist so-called singular controls [27] at which surjectivity does not hold, numerical results show that their impact on the search for optimal controls should be negligible [28].

Another important consequence of controllable Kraus-type evolution for various types of quantum OLC, AFC and RTFC 
is the existence of a special control (e.g., for measurementbased RTFC, a special feedback process $F_{*}$ ) that is optimal for all initial states of the system. Let $\rho(T)$ be the state maximizing the target expectation value: $\operatorname{Tr}[\hat{A} \rho(T)]=$ $\max _{\rho} \operatorname{Tr}(\hat{A} \rho)$, and let the spectral decomposition of this final state be $\rho(T)=\sum_{\alpha=1}^{n} p_{\alpha}\left|u_{\alpha}\right\rangle\left\langle u_{\alpha}\right|$, where $p_{\alpha}$ is the probability to find the system in the state $\left|u_{\alpha}\right\rangle$. For an arbitrary orthonormal basis $\left\{v_{\beta}\right\}$ in the system's Hilbert space, define operators $K_{\alpha, \beta}=p_{\alpha}^{1 / 2}\left|u_{\alpha}\right\rangle\left\langle v_{\beta}\right|$. The Kraus map built from these operators, $\Phi_{*}(\rho)=\sum_{\alpha, \beta=1}^{n} K_{\alpha, \beta} \rho K_{\alpha, \beta}^{\dagger}$ generates evolution $\Phi_{*}[\rho(0)]=\rho(T)$ for all initial states $\rho(0)$ [26]. Therefore, the control that produces the Kraus map $\Phi_{*}$ (e.g., for measurement-based RTFC, the feedback process $F_{*} \in \mathcal{F}$ such that $\Phi_{F_{*}}=\Phi_{*}$ ) will be optimal for all initial states and this control will be robust to variations of the initial system state.

This work shows that the operationally and technologically distinct quantum control approaches of OLC, AFC, and RTFC share, under the condition of Kraus-map controllability, a unified control landscape structure implying two common funda- mental properties. First, all such control schemes are characterized by the absence of landscape traps, with all local extrema being saddles. Second, special controls exist which are universally optimal for all initial states. These findings establish that (1) there are no inherent landscape features hindering attainment of the highest possible control yield and (2) suitable controls can provide broad scale robustness to variations of the initial conditions. Moreover, these properties are valid for any quantum control scheme which produces Kraus-type evolution of the system, including feedback-based and openloop approaches, as well as their combinations. The unification of these seemingly different approaches at a conceptual level (see figures in supplementary material [9]) should, in turn, provide a basis to ultimately unite the currently distinct laboratory realizations of quantum feedback control and openloop control to attain the best performance under all possible conditions.

This work was supported by NSF and ARO.
[1] C. Brif, R. Chakrabarti, and H. Rabitz, New J. Phys. 12, 075008 (2010).

[2] A. P. Peirce, M. A. Dahleh, and H. Rabitz, Phys. Rev. A 37, 4950 (1988); S. Shi and H. Rabitz, J. Chem. Phys. 92, 364 (1990).

[3] N. Timoney et al., Phys. Rev. A 77, 052334 (2008); M. J. Biercuk et al., ibid. 79, 062324 (2009); Y. Silberberg, Annu. Rev. Phys. Chem. 60, 277 (2009); F. Krausz and M. Ivanov, Rev. Mod. Phys. 81, 163 (2009).

[4] R. S. Judson and H. Rabitz, Phys. Rev. Lett. 68, 1500 (1992).

[5] H. Rabitz et al., Science 288, 824 (2000).

[6] A. Assion et al., Science 282, 919 (1998); D. Meshulach and Y. Silberberg, Nature (London) 396, 239 (1998); R. Bartels et al., ibid. 406, 164 (2000); T. Brixner et al., ibid. 414, 57 (2001); J. L. Herek et al., ibid. 417, 533 (2002); R. J. Levis, G. M. Menkir, and H. Rabitz, Science 292, 709 (2001); V. I. Prokhorenko et al., ibid. 313, 1257 (2006); M. P. A. Branderhorst et al., ibid. 320, 638 (2008); T. Brixner et al., Phys. Rev. Lett. 92, 208301 (2004); A. Lindinger et al., ibid. 93, 033001 (2004); T. Laarmann et al., ibid. 98, 058302 (2007); M. Roth et al., ibid. 102, 253001 (2009); P. Nuernberger, G. Vogt, T. Brixner, and G. Gerber, Phys. Chem. Chem. Phys. 9, 2470 (2007).

[7] A. Pechen and H. Rabitz, Phys. Rev. A 73, 062102 (2006); R. Romano and D. D'Alessandro, ibid. 73, 022323 (2006).

[8] R. Vilela Mendes and V. I. Man'ko, Phys. Rev. A 67, 053404 (2003); A. Pechen, N. Ilin, F. Shuang, and H. Rabitz, ibid. 74, 052102 (2006); F. Shuang et al., ibid. 78, 063422 (2008).

[9] See supplementary material at [http://link.aps.org/supplemen tal/10.1103/PhysRevA.82.030101], for more information on quantum control approaches, a hybrid quantum control scheme, and Kraus maps.

[10] V. P. Belavkin, Autom. Remote Control 44, 178 (1983).

[11] H. M. Wiseman, Phys. Rev. A 49, 2133 (1994).

[12] H. Rabitz, M. Hsieh, and C. Rosenthal, Science 303, 1998 (2004); H. Rabitz, T.-S. Ho, M. Hsieh, R. Kosut, and M. Demiralp, Phys. Rev. A 74, 012721 (2006); H. Rabitz, M. Hsieh, and C. Rosenthal, J. Chem. Phys. 124, 204107 (2006); M. Hsieh,
R. B. Wu, and H. Rabitz, ibid. 130, 104109 (2009).

[13] M. Hsieh and H. Rabitz, Phys. Rev. A 77, 042306 (2008); T.-S. Ho, J. Dominy, and H. Rabitz, ibid. 79, 013422 (2009).

[14] T.-S. Ho and H. Rabitz, J. Photochem. Photobiol. A 180, 226 (2006).

[15] K. Moore, M. Hsieh, and H. Rabitz, J. Chem. Phys. 128, 154117 (2008); A. Oza et al., J. Phys. A 42, 205305 (2009).

[16] J. G. Digalakis and K. G. Margaritis, Int. J. Comp. Math. 77, 481 (2001).

[17] J. Roslund and H. Rabitz, Phys. Rev. A 79, 053417 (2009); J. Roslund, O. M. Shir, T. Back, and H. Rabitz, ibid. 80, 043415 (2009).

[18] A. Pechen, D. Prokhorenko, R. B. Wu, and H. Rabitz, J. Phys. A 41, 045205 (2008); R. B. Wu, A. Pechen, H. Rabitz, M. Hsieh, and B. Tsou, J. Math. Phys. 49, 022108 (2008).

[19] K. Kraus, States, Effects and Operations: Fundamental Notions of Quantum Theory (Springer, Berlin, 1983).

[20] A. C. Doherty, S. Habib, K. Jacobs, H. Mabuchi, and S. M. Tan, Phys. Rev. A 62, 012105 (2000).

[21] P. Bushev et al., Phys. Rev. Lett. 96, 043003 (2006); G. G. Gillett et al., ibid. 104, 080503 (2010).

[22] S. Lloyd, Phys. Rev. A 62, 022108 (2000).

[23] R. J. Nelson, Y. Weinstein, D. Cory, and S. Lloyd, Phys. Rev. Lett. 85, 3045 (2000); H. Mabuchi, Phys. Rev. A 78, 032323 (2008).

[24] S. Lloyd and L. Viola, Phys. Rev. A 65, 010101 (2001).

[25] D. A. Lidar, Z. Bihary, and K. B. Whaley, Chem. Phys. 268, 35 (2001).

[26] R. Wu, A. Pechen, C. Brif, and H. Rabitz, J. Phys. A 40, 5681 (2007).

[27] B. Bonnard and M. Chyba, Singular Trajectories and Their Role in Control Theory (Springer, Berlin, 2003).

[28] R. B. Wu, J. Dominy, T.-S. Ho, and H. Rabitz, e-print arXiv:0907.2354. 


\title{
Supplementary material for the manuscript "General unifying features of controlled quantum phenomena"
}

\author{
Alexander Pechen, Constantin Brif, Rebing Wu, Raj Chakrabarti, and Herschel Rabitz \\ Department of Chemistry, Princeton University, Princeton, New Jersey 08544, USA
}

Quantum control approaches

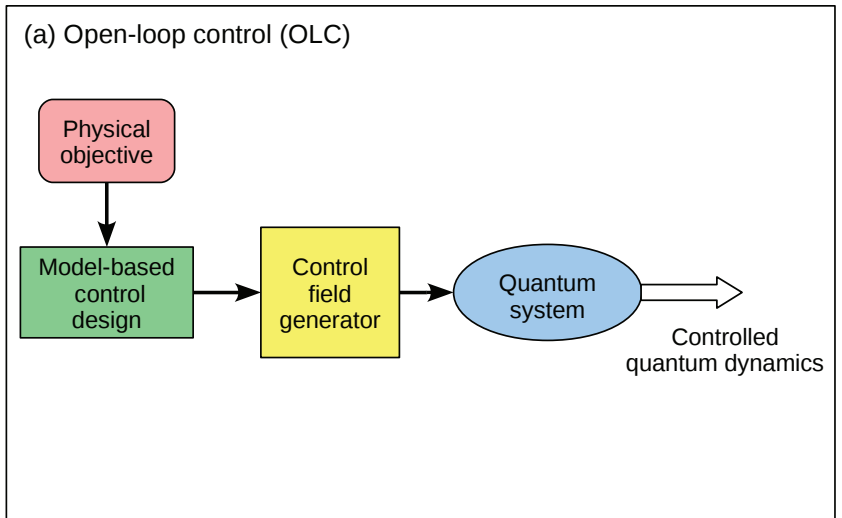

(c) Measurement-based real-time feedback control (RTFC)

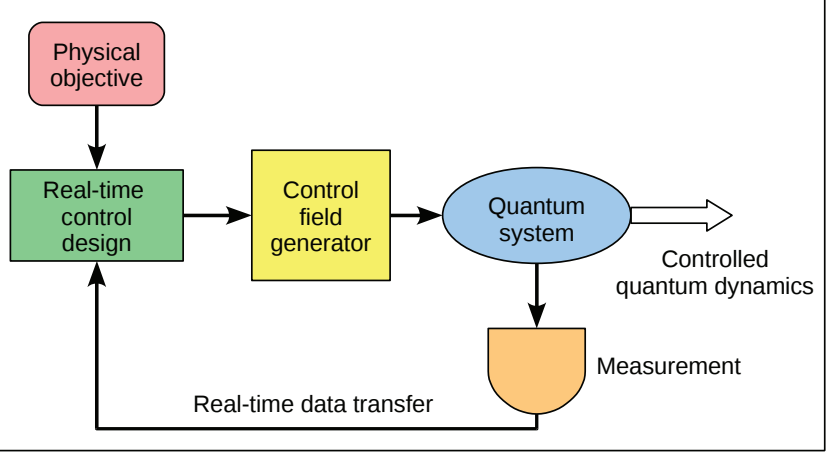

(b) Adaptive feedback control (AFC)

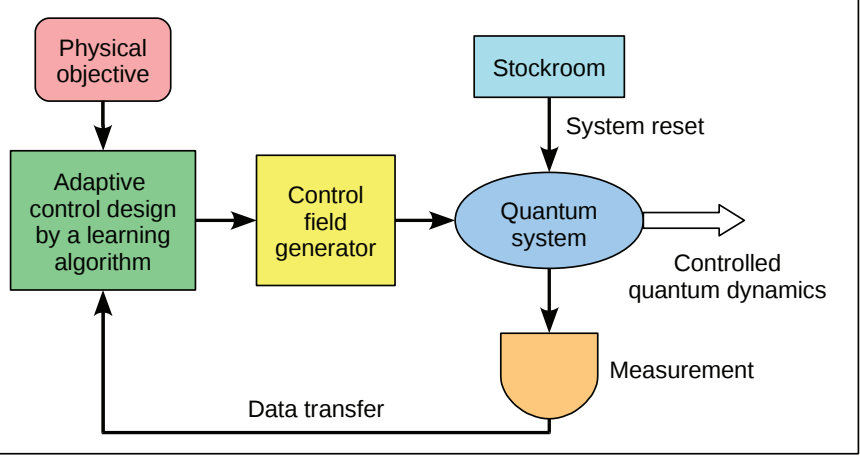

(d) Coherent real-time feedback control (coherent RTFC)

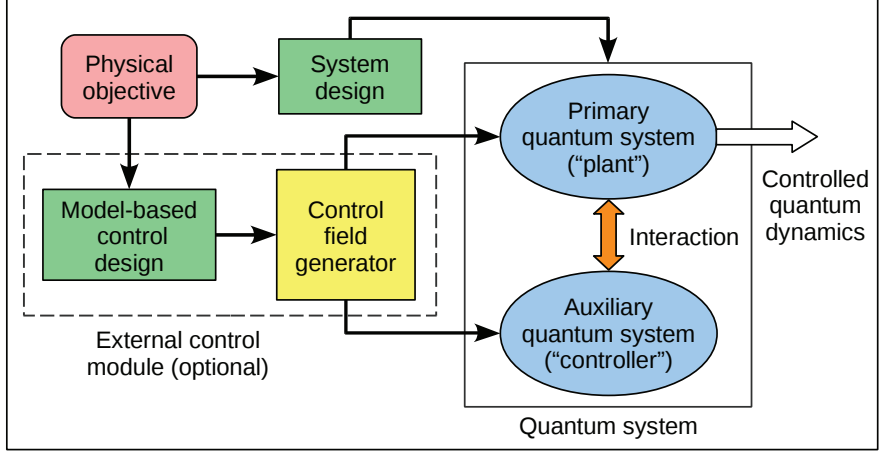

FIG. 1: Outline of different quantum control schemes, all of which aim at achieving a target physical objective by actively manipulating the dynamics of a quantum system. (a) In open-loop control (OLC), a theoretical model of the system dynamics is used to design the control field (e.g., employing optimal control theory). This control design is then directly applied in the laboratory [1,2]. This approach can be satisfactory for some simple systems; however, in more complex situations, the model-based design is usually not optimal for the actual system [3]. (b) In adaptive feedback control (AFC), the objective is measured at each iteration and the result is fed back to a learning algorithm that uses previous observations to propose a new (ideally, better) control [4]. At the end of each trial, after the control is applied and the objective is measured, the system is reset to its initial state for the next iteration. This resetting makes the back action of the measurement irrelevant. In recent years, $\mathrm{AFC}$ has become a routine procedure that is successfully used in many laboratories for control of various quantum phenomena $[3,5,6]$. Of particular importance is the ability of AFC to function in the laboratory in situations when the Hamiltonian of a complex quantum system and its environment are not well known. (c) In the measurement-based type of real-time feedback control (RTFC), measurements are employed to probe the quantum system, and the obtained information is processed classically in real time to select the next control action [7-11]. In this scheme, the system evolution during each feedback iteration is affected not only by the coherent control action (conditioned upon the measurement outcome), but also by incoherent back-action exerted by the measurement. Variations of RTFC with both discrete and continuous measurements were studied [10]. An important factor in considering laboratory implementation of RTFC on the atomic or molecular scale is the issue of loop latency arising due to the fact that many interesting quantum phenomena occur on a time scale which is too short to allow for processing of the measured data in classical controllers based on conventional electronics. (d) In coherent RTFC, the evolution of the primary quantum system (the "plant") is manipulated through its interaction with an auxiliary quantum system (the "controller") [12-14]. The evolution of the composite quantum system which consists of the plant and controller is unitary (assuming that environmental effects are neglected). Optionally, the plant, controller, or both can be also coherently driven by external classical control fields (e.g., designed based on a theoretical model of the composite system dynamics, as in OLC). The optional external control module is shown within the dashed box. While coherent RTFC can overcome the latency issue, the quantum controller itself may require precise engineering to assure quality control performance of the plant. 


\section{A prospective hybrid scheme of quantum control}

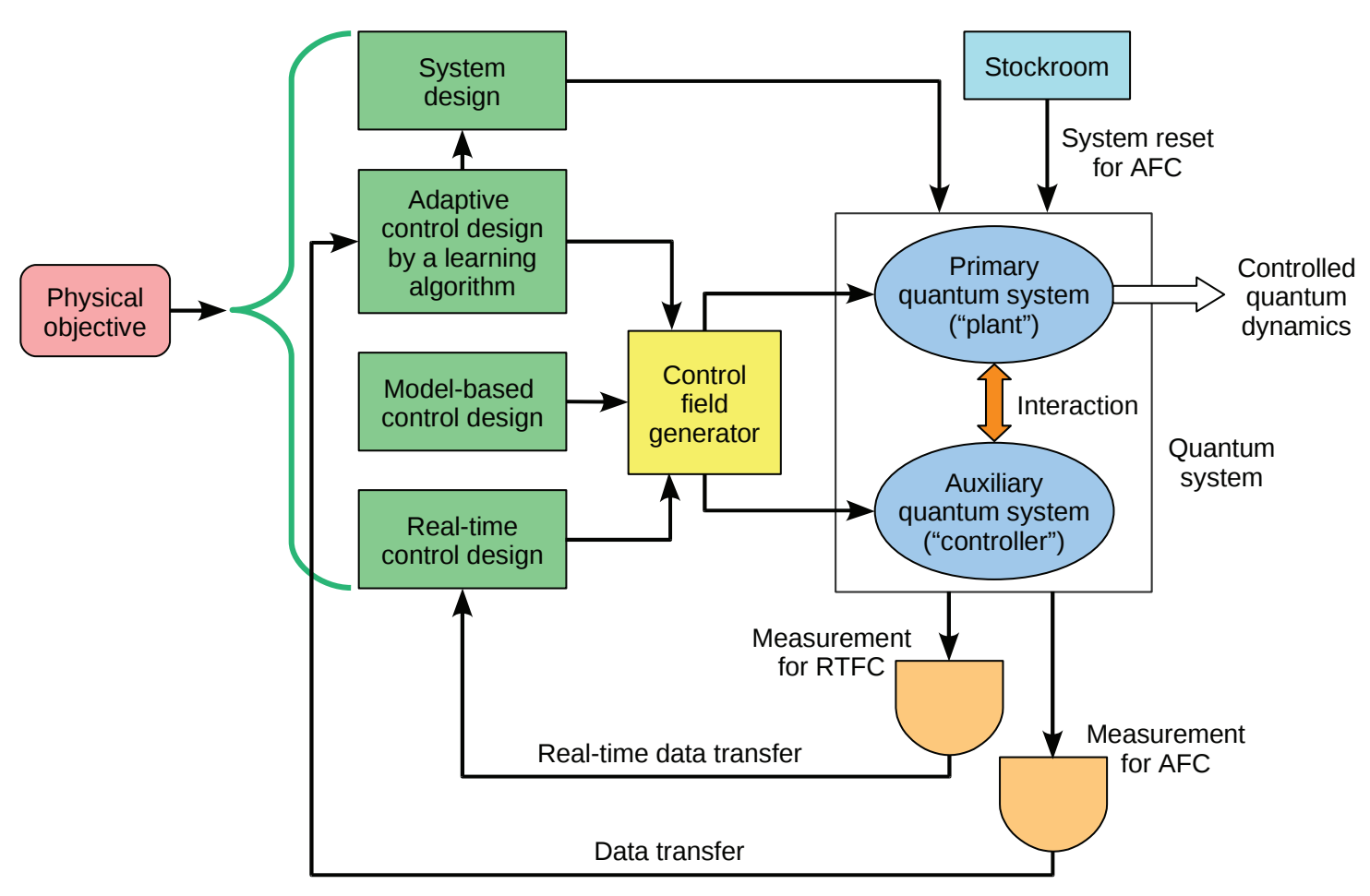

FIG. 2: Outline of a prospective hybrid scheme of quantum control [15]. Since all quantum control approaches, including OLC, AFC, and different types of RTFC, share the same trap-free control landscape topology, it would be possible to consistently maximize the attainment of a target physical objective by utilizing a hybrid control scheme. Such a hybrid scheme would benefit from synergetic contributions of all components tuned to achieve the highest degree of control over the quantum dynamics. In particular, it can incorporate coherent RTFC which employs the interaction of the plant (the primary quantum system) with a quantum controller. Additionally, both the plant and controller can be manipulated by coherently driving them with external classical control fields. In order to improve the effectiveness of external controls, a measurement-based RTFC loop can be implemented, in which the quantum system is probed at each feedback iteration to assess the best next control action. Moreover, quantum measurement back-action can be employed as an additional non-unitary control. The overall performance of the control system can be optimized in the AFC loop, with the measurement of the objective followed, at the end of each trial, by resetting the system to its initial state. AFC can be very useful for optimizing designs and operational conditions of both quantum and classical controllers employed in coherent and measurement-based RTFC, respectively. While model-based theoretical control designs utilized in OLC will typically not be optimal for actual complex quantum systems in the laboratory, they can serve as initial trial fields in the closed-loop optimization as well as play an important role in exploring the feasibility of various control outcomes. The order, in which the components of a hybrid control scheme are implemented, can vary depending on practical circumstances.

\section{Kraus maps}

The density matrix $\rho$ representing the state of an $n$-level quantum system is a positive, unit-trace $n \times n$ matrix. We denote by $\mathcal{M}_{n}=\mathbb{C}^{n \times n}$ the set of all $n \times n$ complex matrices, and by $\mathcal{D}_{n}:=\left\{\rho \in \mathcal{M}_{n} \mid \rho=\rho^{\dagger}, \rho \geq 0, \operatorname{Tr}(\rho)=1\right\}$ the set of all density matrices. A map $\Phi: \mathcal{M}_{n} \rightarrow \mathcal{M}_{n}$ is positive if $\Phi(\rho) \geq 0$ for any $\rho \geq 0$ in $\mathcal{M}_{n}$. A map $\Phi: \mathcal{M}_{n} \rightarrow \mathcal{M}_{n}$ is completely positive (CP) if for any $l \in \mathbb{N}$ the map $\Phi \otimes \mathbb{I}_{l}: \mathcal{M}_{n} \otimes \mathcal{M}_{l} \rightarrow \mathcal{M}_{n} \otimes \mathcal{M}_{l}$ is positive ( $\mathbb{I}_{l}$ is the identity map in $\mathcal{M}_{l}$ ). A CP map is trace-preserving if $\operatorname{Tr}[\Phi(\rho)]=\operatorname{Tr}(\rho)$ for any $\rho \in \mathcal{M}_{n}$. We denote by $\mathcal{K}_{n}$ the set of all CP, trace-preserving maps acting in $\mathcal{M}_{n}$, referred to as Kraus maps or quantum operations [16-18].

[1] R. Fanciulli, A. M. Weiner, M. M. Dignam, D. Meinhold, and K. Leo, Phys. Rev. B 71, 153304 (2005); N. Dudovich, T. Polack, A. Pe'er, and Y. Silberberg, Phys. Rev. Lett. 94, 083002 (2005); Z. Amitay, A. Gandman, L. Chuntonov, and L. Rybak, ibid. 100, 193002 (2008); N. Timoney, V. Elman, S. Glaser, C. Weiss, M. Johanning, W. Neuhauser, and C. Wunderlich, Phys. Rev. A 77, 052334 (2008); M. J. Biercuk, H. Uys, A. P. VanDevender, N. Shiga, W. M. Itano, and J. J. Bollinger, ibid. 79, 062324 (2009). 
[2] H. Stapelfeldt and T. Seideman, Rev. Mod. Phys. 75, 543 (2003); M. Dantus and V. V. Lozovoy, Chem. Rev. 104, 1813 (2004); Y. Silberberg, Ann. Rev. Phys. Chem. 60, 277 (2009); F. Krausz and M. Ivanov, Rev. Mod. Phys. 81, 163 (2009).

[3] C. Brif, R. Chakrabarti, and H. Rabitz, New J. Phys. 12, 075008 (2010).

[4] R. S. Judson and H. Rabitz, Phys. Rev. Lett. 68, 1500 (1992).

[5] A. Assion et al., Science 282, 919 (1998); D. Meshulach and Y. Silberberg, Nature 396, 239 (1998); T. C. Weinacht, J. Ahn, and P. H. Bucksbaum, ibid. 397, 233 (1999); R. Bartels et al., ibid. 406, 164 (2000); T. Brixner, N. H. Damrauer, P. Niklaus, and G. Gerber, ibid. 414, 57 (2001); J. L. Herek, W. Wohlleben, R. J. Cogdell, D. Zeidler, and M. Motzkus, ibid. 417, 533 (2002); R. J. Levis, G. M. Menkir, and H. Rabitz, Science 292, 709 (2001); T. Brixner et al., Phys. Rev. Lett. 92, 208301 (2004); A. Lindinger, C. Lupulescu, M. Plewicki, F. Vetter, A. Merli, S. M. Weber, and L. Wöste, ibid. 93, 033001 (2004); V. I. Prokhorenko, A. M. Nagy, S. A. Waschuk, L. S. Brown, R. R. Birge, and R. J. D. Miller, Science 313, 1257 (2006); T. Laarmann et al., Phys. Rev. Lett. 98, 058302 (2007); M. P. A. Branderhorst, P. Londero, P. Wasylczyk, C. Brif, R. L. Kosut, H. Rabitz, and I. A. Walmsley, Science 320, 638 (2008); M. J. Biercuk, H. Uys, A. P. VanDevender, N. Shiga, W. M. Itano, and J. J. Bollinger, Nature 458, 996 (2009); M. Roth, L. Guyon, J. Roslund, V. Boutou, F. Courvoisier, J.-P. Wolf, and H. Rabitz, Phys. Rev. Lett. 102, 253001 (2009).

[6] H. Rabitz, R. de Vivie-Riedle, M. Motzkus, and K. Kompa, Science 288, 824 (2000); R. J. Levis and H. A. Rabitz, J. Phys. Chem. A 106, 6427 (2002); D. Goswami, Phys. Rep. 374, 385 (2003); T. Brixner and G. Gerber, ChemPhysChem 4, 418 (2003); M. Wollenhaupt, V. Engel, and T. Baumert, Ann. Rev. Phys. Chem. 56, 25 (2005); W. Wohlleben, T. Buckup, J. L. Herek, and M. Motzkus, ChemPhysChem 6, 850 (2005); T. Pfeifer, C. Spielmann, and G. Gerber, Rep. Prog. Phys. 69, 443 (2006); P. Nuernberger, G. Vogt, T. Brixner, and G. Gerber, Phys. Chem. Chem. Phys. 9, 2470 (2007); C. Winterfeldt, C. Spielmann, and G. Gerber, Rev. Mod. Phys. 80, 117 (2008).

[7] V. P. Belavkin, Autom. Remote Control 44, 178 (1983).

[8] H. M. Wiseman and G. J. Milburn, Phys. Rev. Lett. 70, 548 (1993); H. M. Wiseman, Phys. Rev. A 49, 2133 (1994).

[9] A. C. Doherty, S. Habib, K. Jacobs, H. Mabuchi, and S. M. Tan, Phys. Rev. A 62, 012105 (2000); A. Doherty, J. Doyle, H. Mabuchi, K. Jacobs, and S. Habib, in Proceedings of the 39th IEEE Conference on Decision and Control (IEEE, 2000), vol. 1, p. 949.

[10] H. M. Wiseman and G. J. Milburn, Quantum Measurement and Control (Cambridge University Press, Cambridge, UK, 2010).

[11] P. Bushev et al., Phys. Rev. Lett. 96, 043003 (2006); A. J. Berglund, K. McHale, and H. Mabuchi, Opt. Lett. 32, 145 (2007); G. G. Gillett et al., Phys. Rev. Lett. 104, 080503 (2010).

[12] S. Lloyd, Phys. Rev. A 62, 022108 (2000).

[13] M. R. James, H. I. Nurdin, and I. R. Petersen, IEEE Trans. Autom. Control 53, 1787 (2008).

[14] R. J. Nelson, Y. Weinstein, D. Cory, and S. Lloyd, Phys. Rev. Lett. 85, 3045 (2000); H. Mabuchi, Phys. Rev. A 78, 032323 (2008).

[15] R. L. Kosut, R. J. Levis, H. Mabuchi, H. Rabitz, I. A. Walmsley, and E. Yablonovich, "Managing uncertainties in the control of quantum systems," Summary of 2003 Princeton-Oxford Workshop on Control of Quantum Systems (unpublished).

[16] K. Kraus, States, Effects and Operations: Fundamental Notions of Quantum Theory (Springer, Berlin, 1983).

[17] R. Alicki and K. Lendi, Quantum Dynamical Semigroups and Applications (Springer, Berlin, 2007).

[18] M.-D. Choi, Lin. Alg. Appl. 10, 285 (1975). 